\title{
Aplicação das ferramentas da qualidade FMEA e FTA para detecção e controle de patologias em estruturas de concreto armado do Aeroporto Internacional do Recife
}

Title: Application of quality tools FMEA and FTA for detection and control of diseases in reinforced concrete structures from the Recife International Airport

\author{
Severino Virgílio da Silva \\ Escola Politécnica de Pernambuco \\ Universidade de Pernambuco \\ 50.720-001 - Recife, Brasil \\ svirgilio81@gmail.com
}

\author{
Eliana Cristina Barreto Monteiro \\ Escola Politécnica de Pernambuco \\ Universidade de Pernambuco \\ 50.720-001 - Recife, Brasil \\ eliana@poli.br
}

\begin{abstract}
Resumo Com o aumento do número de ocorrências patológicas apresentadas pelas estruturas de concreto armado, conforme levantamentos realizados por diversos autores ao longo os últimos anos, é crescente a demanda por serviços de inspeção e manutenção em tais estruturas no Estado de Pernambuco e em todo o País. No entanto, é importante salientar que a prática de inspeções periódicas ainda não faz parte da cultura da maioria dos usuários, devido à falta de conhecimento da importância da manutenção para garantir a integridade da estrutura. Por isso é necessário a racionalização do processo de manutenção, que pode ser atingida com a utilização de duas ferramentas de gestão da qualidade total combinadas, a análise da árvore de falhas (FTA) e a análise dos Modos de Falhas e seus Efeitos (FMEA), visando a realização de inspeções nas estruturas para a montagem de um banco de dados que permita a hierarquização por nível de risco das patologias detectadas e à criação de planos de ação preventiva e corretiva a fim de diminuir sua ocorrência. Neste trabalho analisou-se as manifestações patológicas encontradas no viaduto de acesso ao Aeroporto Internacional do Recife, oriundas de uma inspeção prévia, foram aplicados os métodos de gestão integrada da qualidade a fim de avaliar os possiveis danos causados à estrutura. A combinação dos métodos permitiu a definição da criticidade das manifestações patológicas pela ótica de suas causas básicas e seus efeitos de maior relevância, configurando-se como uma importante ferramenta para a análise sistêmica de patologias em estruturas de concreto armado.
\end{abstract}

Palavras-Chave: Aeroporto, inspeção, manutenção

\begin{abstract}
With the increasing number of pathological occurrences made by reinforced concrete structures, according to surveys conducted by various authors over the last few years, there is a growing demand for inspection and maintenance services for such structures in the state of Pernambuco and throughout the country. However, it is important to note that the practice of periodic inspections is not yet part of the culture of most users, due to lack of knowledge of the importance of maintenance to ensure the integrity of the structure. So we need to streamlining the maintenance process, which can be achieved with the use of two tools of management of the combined total quality, the analysis of the fault tree (FTA) and the analysis of Failure Mode and Effects (FMEA) in order to carry out inspections on structures for assembling a database that allows the ranking by risk level of the detected gross pathologies and the creation of preventive and corrective action plans in order to reduce its occurrence. In this paper we analyzed the pathological changes found by viaduct access to the Recife International Airport, coming from a preview, the integrated management of quality methods were applied to assess the potential damage caused to the structure. The combination of the methods allowed for the definition of the criticality of the pathological manifestations through the eyes of its root causes and its most relevant effects, setting up as an important tool for systemic analysis of conditions in reinforced concrete structures.
\end{abstract}

Keywords: Airport, inspection, maintenance 


\section{Introdução}

O concreto armado, assim como os demais materiais presentes em uma edificação, não pode ser considerado eterno pois necessita de manutenção periódica para garantir a sua vida útil. A falta de manutenção ou a falha na execução dos reparos necessários abre precedente para a ocorrência de manifestações patológicas de relativa intensidade, que por sua vez é capaz de gerar custos elevados para a reabilitação.

Antes do início do processo de reabilitação de uma estrutura de concreto é fundamental identificar a origem do processo de degradação. Muitas vezes, apenas a recuperação do concreto ou do aço não é suficiente para reabilitar completamente a estrutura, em virtude do ambiente e microclima a qual a estrutura esteja inserida, sendo necessário avaliar a necessidade de aplicação de tratamentos superficiais protetores [1].

Passados mais de 10 anos desde sua revitalização em 2004, o Aeroporto Internacional do Recife/GuararapesGilberto Freyre, bem como as edificações dessa idade, apresenta manifestações patológicas em algumas de suas estruturas de concreto. Mesmo estando inserido num ambiente urbano, considerado de classe de agressividade moderada, há a necessidade da realização de inspeções periódicas em todas a estruturas que o compõem, devido ao grande fluxo de veículos no seu entorno, responsáveis pelo incremento de $\mathrm{CO} 2$ na atmosfera e a relativa proximidade da zona marinha.

Assim, o presente trabalho visa utilizar ferramentas de gestão da qualidade para auxiliar na deteç̧ão e controle de possíveis patologias em estruturas de concreto armado, constatadas por meio de inspeções na estrutura do viaduto de acesso ao Aeroporto Internacional do Recife.

\subsection{Aeroporto Internacional do Reci- fe/Guararapes-Gilberto Freyre}

É o principal terminal aeroportuário do estado de Pernambuco. Está localizado no bairro da Imbiribeira, Zona Sul do Recife, na divisa com o município de Jaboatão dos Guararapes. O Aeroporto Internacional do Recife é um dos três aeroportos do estado de Pernambuco que atualmente possui operações regulares de transporte de passageiros, juntamente com o Aeroporto de Petrolina e com o Aeroporto de Fernando de Noronha.

O Aeroporto Internacional do Recife opera voos domésticos e internacionais 24 horas por dia e seu nome é uma alusão ao fato histórico da Batalha dos Guararapes, ocorrida no período colonial brasileiro sobre os morros de mesmo nome, situados em sua lateral oeste. Sua construção antecede a II Guerra Mundial, sendo que o conflito serviu para melhorar a estrutura da Base Aérea do Recife e, consequentemente, do próprio aeroporto. No final da década de 40, o Recife passou a ter grande importância no tráfego aéreo, devido sua posição estratégica [2].

\section{Metodologia de Inspeção}

As vistorias em estruturas de concreto podem ser realizadas utilizando diversas técnicas de identificação, de análise e acompanhamento das manifestações patológicas. Por conseguinte, as metodologias empregadas são de vital importância para a realização desta atividade, a fim de proporcionar mecanismos que permitam a caracterização das manifestações patológicas e a proposição de atividades preventivas.

Conforme SILVA [3], pode-se afirmar que os procedimentos de vistoria adquirem um papel fundamental, visto que admitem uma precoce análise que permite grande precisão nos serviços de recuperação, assim como proporciona satisfatórios prognósticos no que concerne à durabilidade. A metodologia empregada neste trabalho seguirá o procedimento de vistorias de estruturas de concreto conforme discriminado na Norma DNIT 010/2004 PRO [4].

É importante mencionar que não foi possível utilizar instrumentos especiais ou realizar ensaios de laboratório. Contudo, a vistoria realizada se mostrou eficaz, pois serviu de base para aplicação das técnicas de análise de confiabilidade aqui estudadas.

\section{Caracterização da estrutura}

$\mathrm{O}$ viaduto indicado, Figura 1, teve sua construção concluída em 2002, está implantado sobre uma via de acesso terminal de passageiros, possuindo pista dupla com $8,60 \mathrm{~m}$ de largura, possuindo cerca de $380,00 \mathrm{~m}$ de comprimento, que dá acesso ao setor de embarque, localizado no primeiro pavimento do terminal de passageiros.

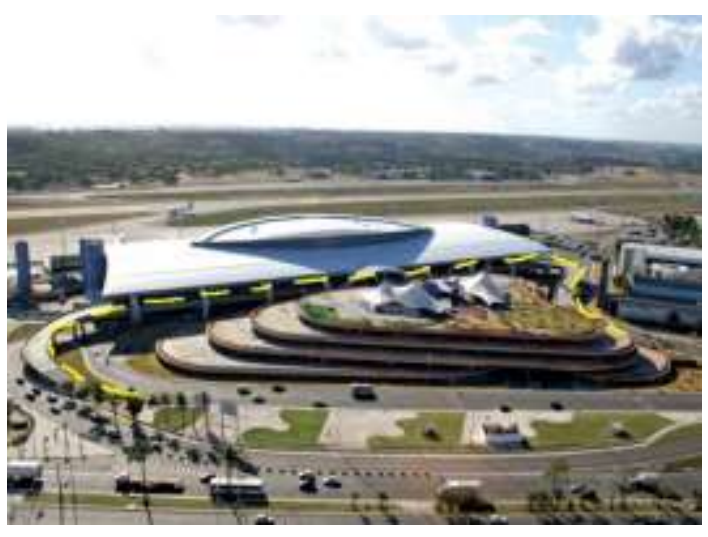

Figura 1: Indicação da área estudada. [Fonte: INFRAERO, 2002 - Adaptado] 
O trecho central é composto por 8,0 (oito) vãos simétricos contendo extensão de $30,00 \mathrm{~m}$, perfazendo uma extensão de $240,00 \mathrm{~m}$. A superestrutura do vão central foi executada em concreto protendido com cabos tipo CP 190 RB Ø 12,7 mm. A Figura 2 exibe o trecho central.

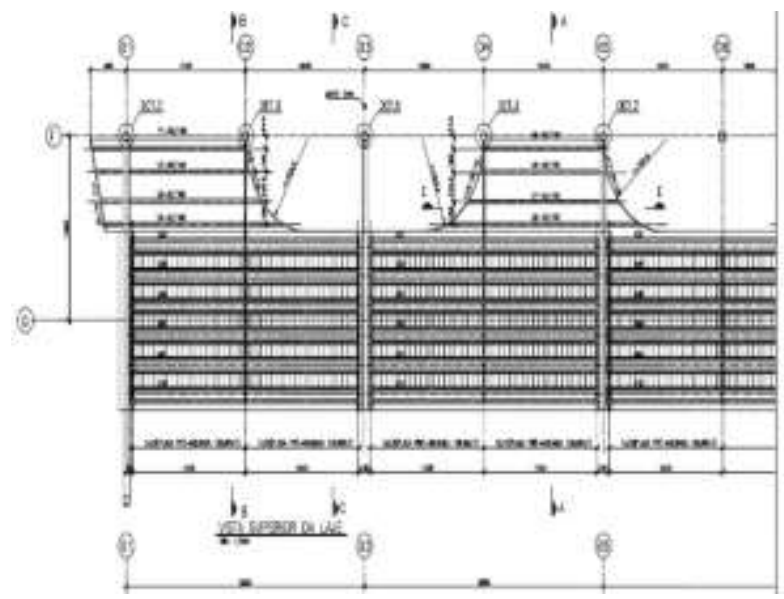

Figura 2: Vista superior do trecho central do tabuleiro. [Fonte: INFRAERO, 2002 - Adaptado]

É composta por 02 (duas) rampas de acesso adjacentes ao trecho central com vão central de $3,60 \mathrm{~m}$, contemplando uma extensão de aproximadamente $70 \mathrm{~m}$. desta forma, o comprimento total do viaduto é de cerca de $380,00 \mathrm{~m}$, como visto na Figura 3.

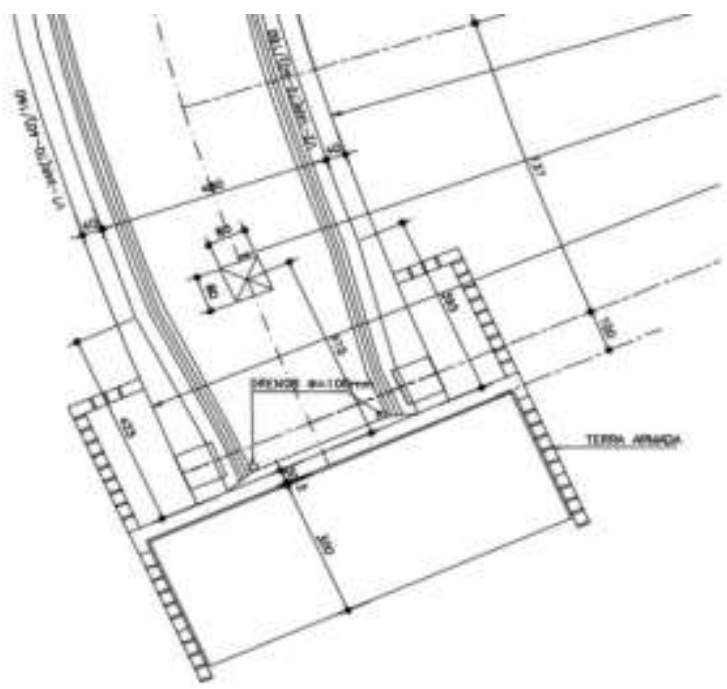

Figura 3: Rampa de acesso, lado esquerdo.

[Fonte: INFRAERO, 2002 - Adaptado]

A seção transversal do viaduto possui largura total de $8,60 \mathrm{~m}$, figura 4 , sendo composto por piso em concreto e guarda-corpos laterais em concreto armado.

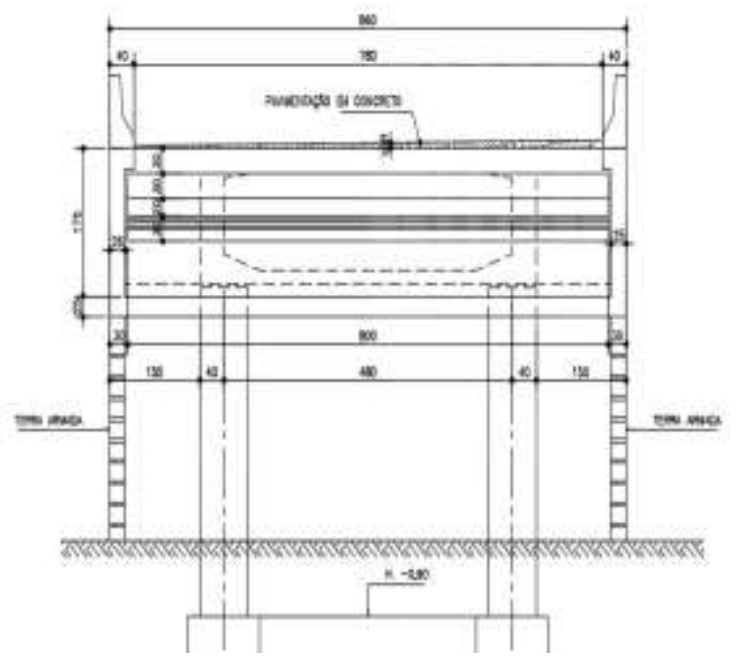

Figura 4: Seção transversal.

[Fonte: INFRAERO, 2002 - Adaptado]

A mesoestrutura foi executada em concreto armado, caracteriza-se por apresentar 08 (oito) pilares que apoiam as rampas de acesso contendo dimensões e alturas variáveis. Cada um dos pilares se apoia em blocos de concreto armado de dimensões também variáveis, e estes em estacas metálicas. Existem ainda os pilares localizados nas extremidades esquerda e direita possuindo dimensões mais robustas que os demais. As Figuras 5 e 6 apresentam detalhes dos pilares e os blocos de fundação.

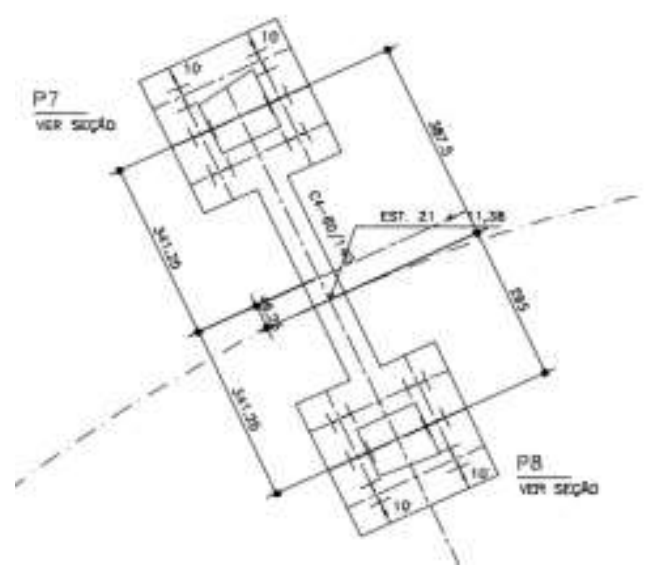

Figura 5: Fundação do viaduto.

[Fonte: INFRAERO, 2002 - Adaptado]

A infraestrutura foi executada em concreto armado. A Figura 6 apresenta o detalhamento de um bloco de fundação da estrutura em questão. 


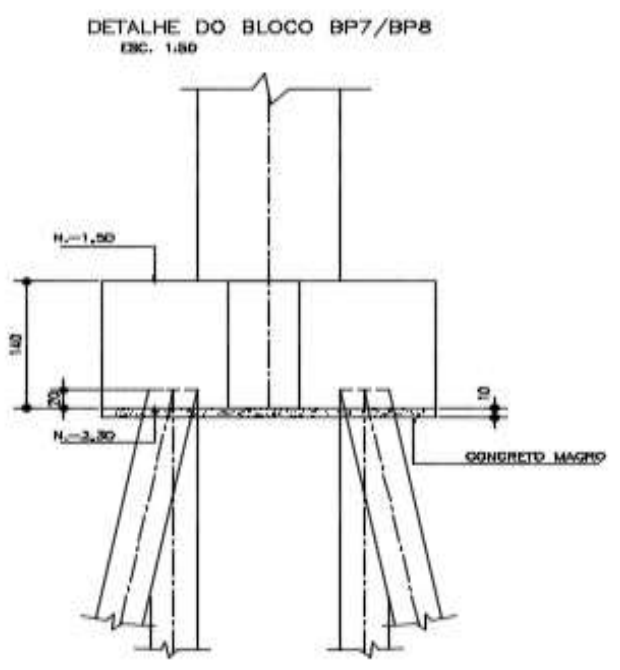

Figura 6: Detalhe do bloco de fundação.

[Fonte: INFRAERO, 2002 - Adaptado]

Em relação aos aparelhos de apoio, estes são do tipo neoprene fretado e situam-se sobre os pilares das rampas de acesso possuindo as dimensões conforme projeto, conforme a Figura 7.

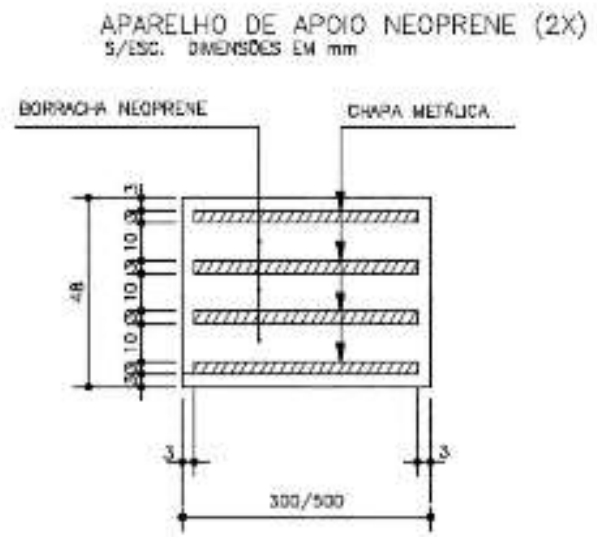

Figura 7: Fundação do viaduto. [Fonte: INFRAERO, 2002 - Adaptado]

\section{Manutenção como garantia da vida útil}

As patologias podem ocorrer numa estrutura tanto na fase de construção, como durante o período de uso. Segundo AZEVEDO [5], as condições que a estrutura apresenta para a ocorrência das ações deletérias podem decorrer de inadequações de projeto, falhas construtivas, falta de manutenção da estrutura ou utilização inadequada, ou ainda a ocorrência de fenômenos naturais imprevisíveis.
Para garantir a adequação das edificações ao uso, a manutenção atualmente configura-se como um instrumento de gestão que deve ser encarado com visão estratégica, ou seja, planejado, previsto e bem executado em todas as suas diretrizes.

Segundo a NBR 5674 [6] - Manutenção de Edificações: Procedimento, as edificações "são construídas para atender seus usuários por muitos anos, e ao longo deste tempo de serviço devem apresentar condições adequadas ao uso que se destinam, resistindo aos agentes ambientais e de uso que alteram suas propriedades técnicas iniciais".

\subsection{O conceito de disponibilidade}

A disponibilidade está intrinsecamente ligada à diminuição da probabilidade de falhas e defeitos na medida em que, com o aumento da capacidade de um item executar uma função entende-se que este estará menos suscetível a problemas. Por sua vez, tais características levam a uma diminuição com custos durante a vida útil do mesmo, uma vez que, aumentados os fatores de proteção, diminui a necessidade de reparos e, por consequência, os gastos periódicos.

Segundo a NBR 5462 [7], disponibilidade pode ser descrita como: "Capacidade de um item estar em condições de executar uma certa função em um dado instante ou durante um intervalo de tempo determinado, levandose em conta os aspectos combinados de sua confiabilidade, mantenabilidade e suporte de manutenção". supondo que os recursos externos requeridos estejam assegurados".

A ABNT NBR 6118: 2014 [8], define os critérios gerais que devem ser seguidos no desenvolvimento de projetos de estruturas de concreto armado e protendido, incluindo os requisitos relacionados com a qualidade da estrutura, enquadrando-as em três grupos distintos: capacidade resistente, desempenho em serviço e durabilidade.

O projetista deverá avaliar também as condições ambientais a que estrutura estará exposta, esta mesma norma classifica o ambiente em quatro classes de agressividade, indicando os limites relativos a relação água/cimento, resistência característica à compressão e cobrimento.

\section{Análise estruturada de falhas e o aumento da confiabilidade}

Para a otimização do processo de manutenção das estruturas, deve-se ter conhecimento das possíveis falhas existentes e potenciais nos sistemas dos mesmos. Uma abordagem racionalizada do processo de manutenção deve ser realizada, criando uma rotina de inspeções.

Tais inspeções, além de detectarem patologias exis- 
tentes, devem ser capazes de listar todas as possíveis causas de cada uma das patologias a fim de que, através da relação causa e efeito, seja possível combater também falhas potenciais. Para tanto, sugere-se o uso de ferramentas da gestão da qualidade total baseada na análise de falhas de um sistema. Deste modo, procura-se definir uma hierarquia de gravidade das patologias encontradas nas estruturas de concreto e explanar suas causas básicas a fim de otimizar custos de manutenção corretiva e criar uma rotina racional de combate a falhas e defeitos nessas estruturas.

A análise de falhas consiste em um segmento da chamada Engenharia de Confiabilidade que visa diminuir a probabilidade de ocorrência de defeitos através da estruturação e planificação de manutenções preventivas e preditivas de acordo com os modos de falha predominantes no sistema e a análise dos riscos representativos ao mesmo, assegurando o controle de suas causas fundamentais identificadas para cada modo.

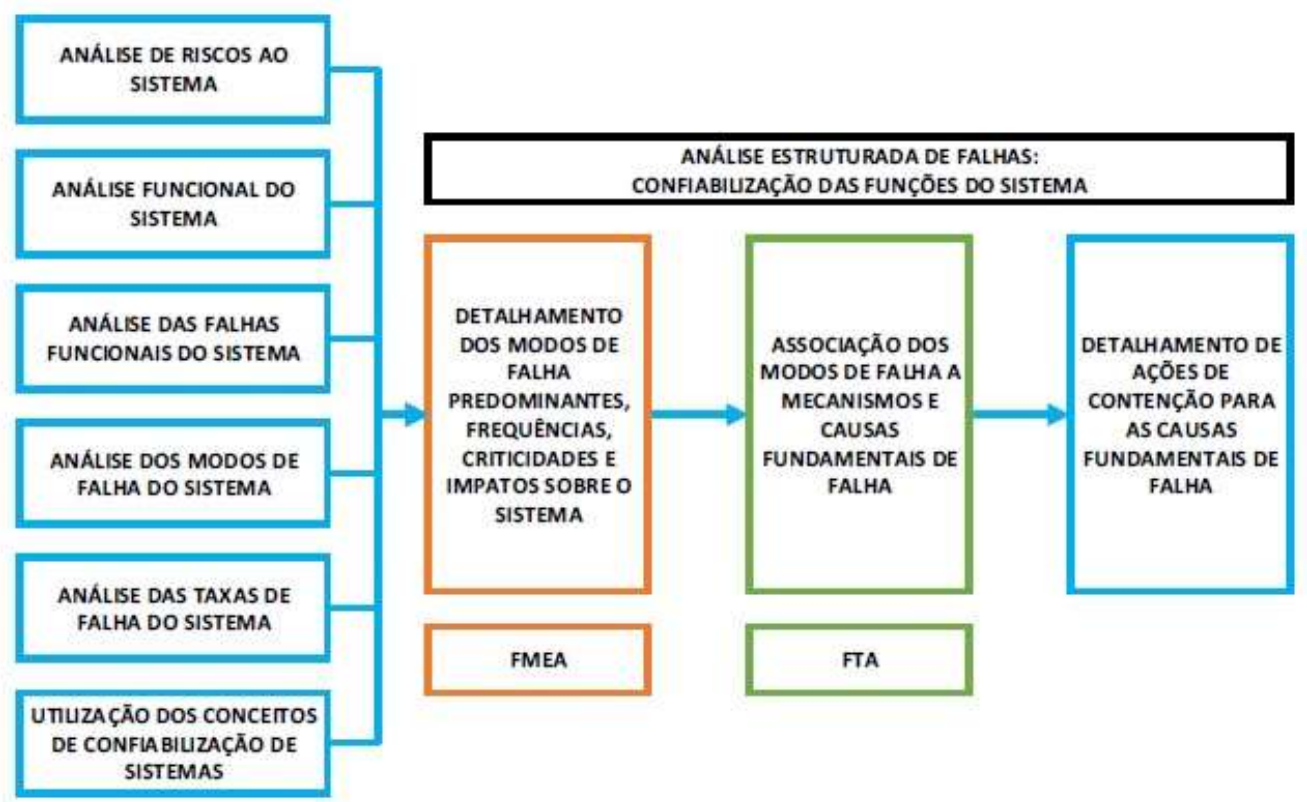

Figure 8: Confiabilização das funções de um sistema através da Análise de Falhas Fonte: Pinto, 2004. (Adaptado)

\subsection{FMEA (Failure Modes And Effects )}

De acordo com STAMATIS [10] "O FMEA (Failure Mode and Effect Analysis) é uma técnica de engenharia utilizada para definir, identificar e eliminar falhas conhecidas ou potenciais, de sistemas, projetos, processos e/ou serviços, antes que estas atinjam o cliente".

A NBR 5462/1994 [7] também define FMEA em seu texto: "Método qualitativo de análise de confiabilidade que envolve o estudo dos modos de panes que podem existir para cada subitem, e a determinação dos efeitos de cada modo de pane sobre os outros subitens e sobre a
PINTO [9], descreve a aplicação da análise de falhas no aumento da confiabilidade dos subsistemas a partir do uso de ferramentas de gestão da qualidade na determinação de risco e causas fundamentais de modos de falha.

$\mathrm{O}$ autor sugere que um processo de análise estruturada de falhas pode ser realizado para aumentar a confiabilidade de sistemas, baseada em atividades prévias de análises de riscos e funcionamento do mesmo. A partir dessa definição, pode-se detalhar falhas predominantes, sua frequência de ocorrência, seus impactos e níveis de criticidade (fatores que podem surgir como produtos do emprego da ferramenta FMEA), e posterior associação das falhas a suas causas fundamentais (aplicação de FTA), para, enfim, detalhar planos de ação de contenção das causas a fim de que as falhas não venham a ocorrer, diminuindo sua frequência e, consequentemente, aumentando a confiabilidade do sistema como um todo. Tais etapas são evidenciadas na Figura 8. 


\subsection{FTA (Fault Tree Analisys)}

O FTA, ou Método de Análise da Árvore de Falhas, é uma das ferramentas empregadas na gestão da qualidade de diversos projetos e processos em empresas, seja na sua fase de implementação, seja em sua execução ou ainda na manutenção da qualidade na fase de uso dos produtos.

Para HELMAN \& ANDERY [11], 1995, o método pode ser definido como o procedimento capaz de "apresentar uma perfeita inter-relação funcional entre uma falha e suas possíveis causas, (...), elaboração de uma árvore associada a cada uma das falhas principais (ou mais comuns) que podem acontecer em um dado equipamento. (...) As árvores de falhas finalizam-se com as possíveis causas de cada problema, e se completam com um plano de ação para sua solução."

O FTA aplicado à manutenção de estruturas, pode ter ação na otimização do processo, na medida em que cria base de dados das falhas, defeitos detectados e suas causas, fator que pode ser utilizado para futuras manutenções e percepção mais nítida do comportamento dos sistemas da edificação ao longo do uso.

Por ser um método gráfico, ainda demonstra visualmente qual seria um caminho crítico de manutenção a ser seguido e quais os efeitos de aplicação dos planos de ação na eliminação ou prevenção de determinada falha para outras falhas consequentes e nos sistemas como um todo.

\section{3 Árvore de falhas}

Segundo SIQUEIRA [12]a árvore de falha é uma ferramenta de análise de cima para baixo (top-down), que se inicia pela identificação de um evento indesejável, chamado de evento raiz, tal como uma falha funcional, prosseguindo pela determinação de todas as maneiras possíveis dela ocorrer. $\mathrm{O}$ autor informa ainda que a análise é conduzida determinando-se como o evento raiz pode ser causado por eventos individuais ou combinação de falhas em níveis inferiores. Para a construção destes diagramas, diversos conectores lógicos e eventos podem ser utilizados, sendo alguns destes relacionados abaixo na Tabela 1.

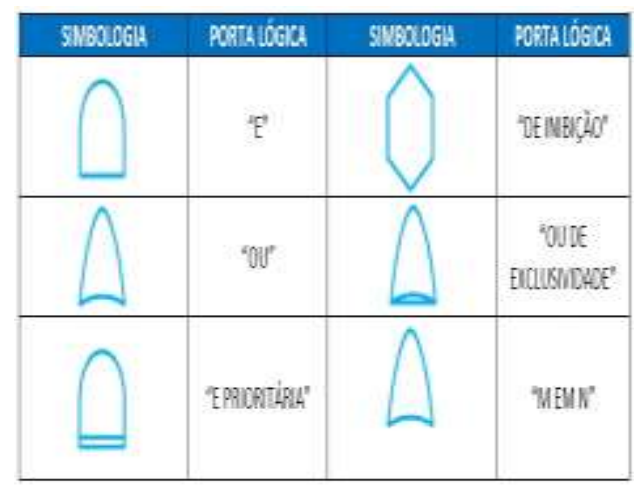

Tabela 1: Conectores lógicos. Fonte: Autor, 2015.
No primeiro momento o responsável pela execução da estrutura deverá diagnosticar as causas dos problemas e os fatores que possam ter contribuído para o seu surgimento. Após entrega e uso, as patologias são constatadas pelos usuários.

Eventos são definidos como as falhas e suas causas, indo de causas básicas e seus efeitos, partindo do denominado evento de topo, que é, em questão, a patologia encontrada da qual se procuram as causas possíveis.

Os eventos diferem em sua simbologia segundo sua significação dentro da estrutura do FTA. Estes podem ser: Retângulo, Círculo, Diamante, Casa dos Eventos ou Triângulo de Transferência.

\section{Resultados Encontrados}

\subsection{Método integrado de FMEA e FTA para detecção e controle de patologias em es- truturas de concreto armado}

Visando maior aproveitamento das ferramentas de gestão da qualidade total para implementação em manutenção de estruturas de concreto que necessitem desempenho satisfatório por longo período de tempo, como é o caso das estruturas que compõem um aeroporto, julga-se mais interessante realizar a combinação dos métodos FMEA e FTA para construção de uma estrutura concisa de avaliação e inspeção que resulte em menores gastos.

O método combinado busca aliar os efeitos dos modos de falha determinados, no caso, as patologias nas estruturas de concreto, às suas causas e efeitos, bem como hierarquizá-las de forma a nortear o desenvolvimento de uma manutenção mais racionalizada.

O método FMEA norteará o processo, sendo o produto final básico deste o formulário demonstrado. A Árvore de Falhas será utilizada para determinar as causas básicas de cada modo de falha encontrado na inspeção e auxiliando na determinação dos índices necessários à hierarquização dos modos de falhas apresentados.

\subsection{Método integrado de FMEA e FTA para detecção e controle de patologias em es- truturas de concreto armado}

Para fins práticos, serão consideradas patologias encontradas em inspeção técnica visual da estrutura analisada, com enfoque nos pontos mais críticos.

A utilização de equipamentos de inspeção em estruturas de concreto é indispensável para o diagnóstico preciso das manifestações patológicas existentes, além da necessidade de possíveis análises laboratoriais, contudo não 
fazem parte desta inspeção realizada, por não ser possível sua implementação no decorrer deste trabalho, sendo recomendadas quando da necessidade de uma melhor caracterização da manifestação patológica.

A partir da identificação de todos os tipos de falhas encontrados, serão determinadas suas causas básicas, as quais serão objetos do restante da análise. Para tal, utiliza-se como subprocesso a confecção das árvores de falhas para cada modo encontrado.

\subsection{Identificação dos tipos de falhas e seus defeitos}

Após a coleta de dados em inspeção e de outras informações disponíveis em registros, faz-se a identificação das patologias encontradas e levantadas, para classificação dos Modos de Falha do método empregado.

As patologias serão classificadas segundo bibliografia acerca do tema e descritas de maneira mais clara possível, de acordo com a sua constatação quando da vistoria realizada.

A partir da identificação de todos os tipos de falhas encontrados, serão determinadas suas causas básicas, as quais serão objetos do restante da análise. Para tal, utiliza-se como subprocesso a confecção das árvores de falhas para cada modo encontrado.

\subsection{Inspeção: coleta de amostras das falhas existentes}

A partir da coleta de dados, faz-se uma lista de verificação prévia contendo elementos passíveis de análise após a vistoria.

A metodologia empregada neste trabalho seguirá o procedimento de vistorias de estruturas de concreto conforme discriminado na Norma DNIT 010/2004 - PRO [4], sendo complementada com a utilização de uma planilha de verificação para vistoria, reproduzida na Tabela 2.

\begin{tabular}{|c|c|c|c|c|}
\hline \multicolumn{5}{|c|}{ INSPEÇÃO DO VIADUTO DE ACESSO AO AEROPORTO INTERNACIONAL DO RECIFE } \\
\hline \multicolumn{5}{|c|}{ NÍVEL DE INSPEÇÃO DEFINIDO A PARTIR DA NORMA DO DNIT 010/2004 - PRO } \\
\hline DATA: & $29 / 06 / 2015$ & & RESPONSÁVEL: & SEVERINO VIRGILIO DA SILVA \\
\hline ITEM & DESCRIÇÃO & AVALIAÇÃO & FOTо & CAUSA APARENTE \\
\hline 1 & Manifestação patológica 1 & $\begin{array}{l}\text { Avaliação a partir da } \\
\text { inspeção visual }\end{array}$ & $\begin{array}{l}\text { IIIIIIIIIIIIIIIIIIII } \\
\text { Foto } 01\end{array}$ & Causa aparente determinada por avaliação prévia \\
\hline 2 & Manifestação patológica 2 & $\begin{array}{l}\text { Avaliação a partir da } \\
\text { inspeção visual }\end{array}$ & $\begin{array}{l}1111111111111111111111 \\
\text { Foto } 02\end{array}$ & Causa aparente determinada por avaliação prévia \\
\hline 3 & Manifestação patológica 3 & $\begin{array}{l}\text { Avaliação a partir da } \\
\text { inspeção visual }\end{array}$ & Foto 03 & Causa aparente determinada por avaliação prévia \\
\hline
\end{tabular}

Tabela 2: Confiabilização das funções de um sistema através da Análise de Falhas Fonte: Pinto, 2004. (Adaptado)

\subsection{Confecção das árvores de falhas}

A confecção das Árvores de Falhas segue padrão descrito para o método FTA de forma simplificada. Inicialmente, faz-se a determinação dos Eventos de Topo de cada árvore. Para o caso, o Evento de Topo corresponderá ao Modo de Falha identificado pela análise dos dados da vistoria. Cada modo será tratado em uma árvore distinta, a fim de que suas causas básicas sejam completamente identificadas e a relação entre as mesmas determinada. Para exemplificar foi confeccionada a árvore de falhas para a patologia denominada como "Trincas em estruturas de concreto", Figura 9.
Após a confecção das árvores de falhas das patologias exibidas na vistoria da estrutura analisada, torna-se possível o preenchimento do formulário FMEA, Tabela 3, relacionando as causas e os efeitos de cada falha encontrada, bem como estimando o grau de risco que este dano causa a estrutura. 


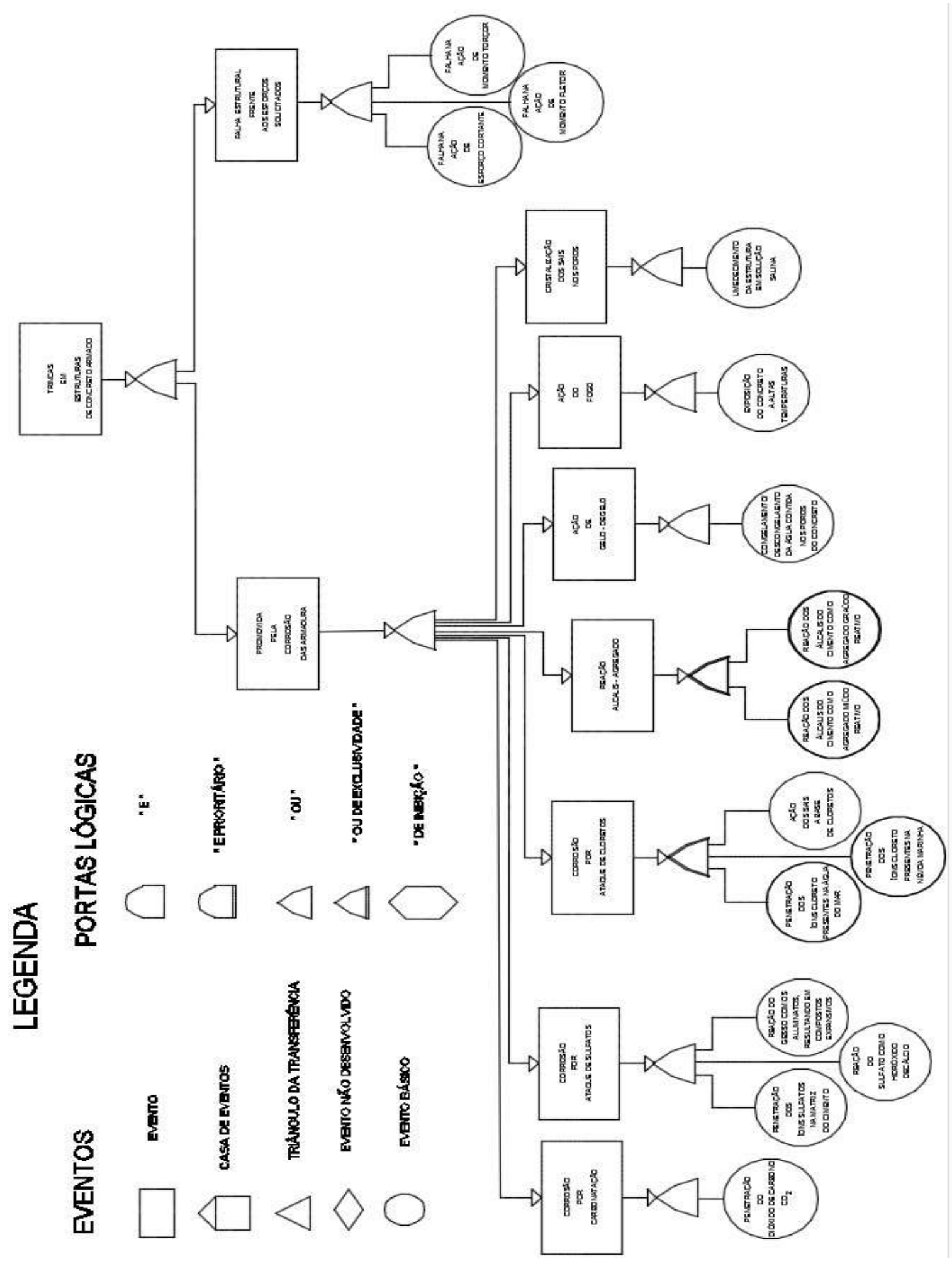

Figure 9: Árvore de falhas para trincas em estruturas de concreto armado. 


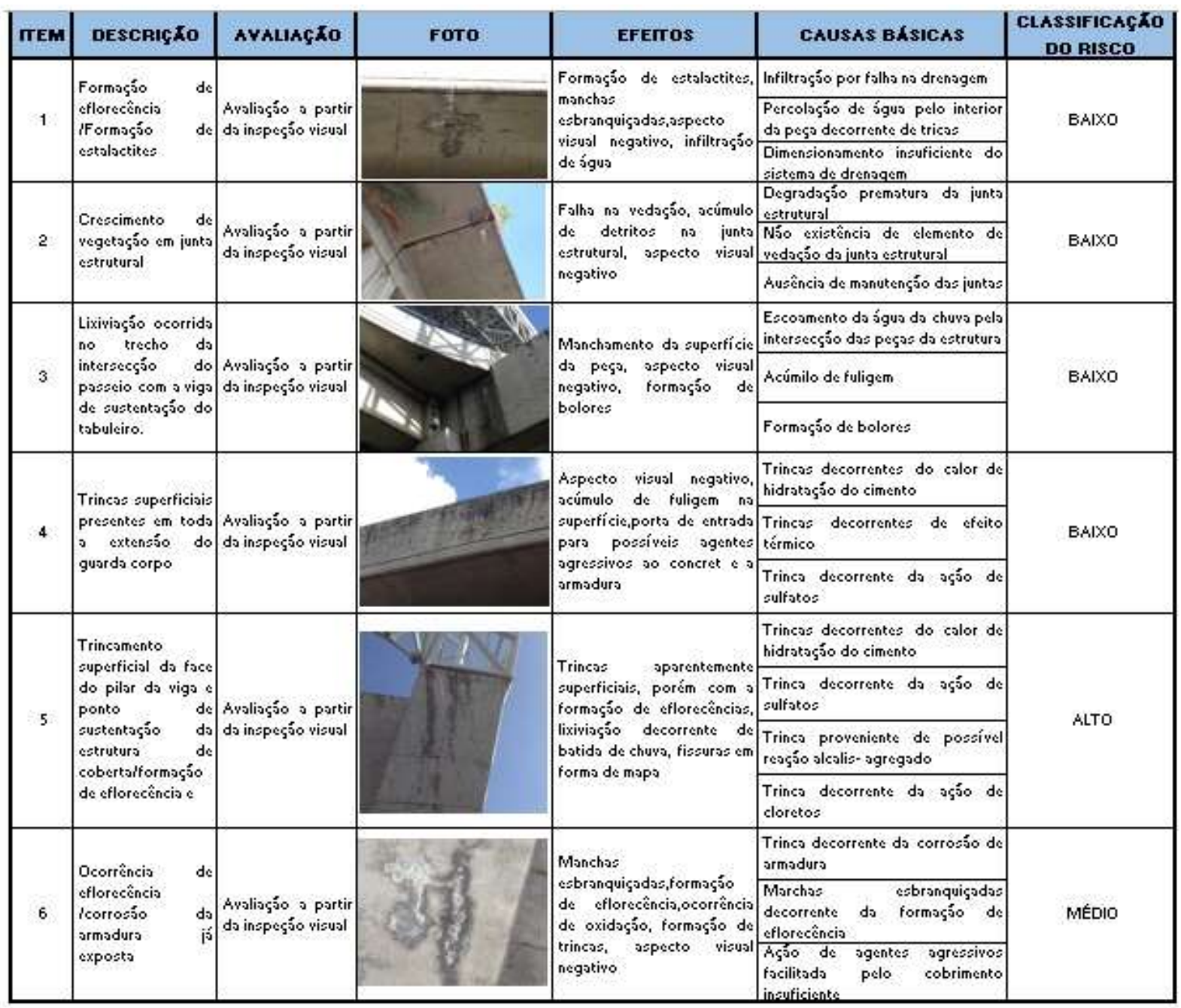

Tabela 3: Formulário FMEA das manifestações patológicas do viaduto de acesso ao Aeroporto Internacional do Recife

\section{Considerações Finais}

As recomendações para correção e erradicação das manifestações patológicas levantadas bem como os planos de ação passíveis de aplicação para um sistema de gestão da manutenção eficiente são tratados como sugestões para trabalhos futuros.

O método empregado foi desenvolvido pelo autor com base na combinação de duas ferramentas de gestão da qualidade orientadas para a análise de falhas e defeitos, FMEA e FTA. A combinação dos métodos permitiu a definição da criticidade das manifestações patológicas pela ótica de suas causas básicas e seus efeitos de maior relevância, configurando-se como uma importante ferramenta para uma análise sistêmica de patologias em estruturas de concreto armado.

$\mathrm{O}$ método permite ainda que ocorrida uma patologia, possa se ter ciência de seus principais efeitos de forma clara e objetiva, bem como suas causas básicas. A ação dos mantenedores deverá ser realizada de forma que gere o bloqueio da manifestação patológica, minimizando também os efeitos já produzidos.

O método pode ser completado com a proposição de planos de ação corretiva e preventiva para os itens identificados na vistoria, a fim de que possa ser definido um padrão de ação para cada patologia. Desta forma, quando o profissional se deparar com uma manifestação patológica, saberá as ações a serem tomadas para seu combate.

Considera-se a aplicação do método desenvolvido um fator inerente à melhoria do processo de manutenção predial, tendo em vista a busca por otimização de resultados e de custo. Com a racionalização desta atividade, os benefícios são diversos, incluindo a diminuição de custos com correções inesperadas, possibilitando planejar as ações de manutenção. 


\section{Referências}

[1] TIMERMAN, JULIO. Reabilitação e reforço de estruturas de concreto. In: ISAIA, Geraldo C. (Ed). Concreto: Ensino, Pesquisa e Realizações. v. 2. São Paulo, SP: IBRACON, 2011. Cap. 33, p.1175 - 1209.

[2] INFRAERO.WWW.infraero.gov.br. http://www.infraero.gov.br/index.php/aeroportos/ pernambuco/aeroporto-internacional-dorecife.html, Ago. 2015.

[3] SILVA, C.J.G. Análise da origem de problemas patológicos em passarela estaiada para pedestres do $\mathrm{km}$ 53,8 DA BR-101/PE. Monografia Pósgraduação em estruturas de concreto e fundações. Recife,2014.

[4] DEPARTAMENTO NACIONAL DE INFRAESTRUTURA DE TRANSPORTES.- DNIT 010/2004 - PRO: Inspeções em pontes e viadutos de concreto armado e protendido - Procedimento. Rio de Janeiro, 2004.

[5] AZEVEDO, MINOS TRICOLI. Patologia das estruturas de concreto. In: ISAIA, Geraldo C. (Ed). Concreto: Ensino, Pesquisa e Realizações. v. 2. São Paulo, SP: IBRACON, 2011. Cap. 31, p.1095 - 1128.

[6] ASSOCIAÇÃO BRASILEIRA DE NORMAS TÉCNICAS. NBR 5674: Manutenção de edificações - Requisitos para o sistema de gestão de manutenção. Rio de janeiro, 2012.

[7] BRASIL. ABNT - Associação Brasileira de Normas Técnicas. NBR 5462:1994: Confiabilidade e Mantenabilidade. 1994.

[8] ASSOCIAÇÃO BRASILEIRA DE NORMAS TÉCNICAS. NBR 6118: Projeto de estruturas de concreto - Procedimento. Rio de janeiro, 2014.

[9] PINTO, Luis Henrique Terbeck. Análise de Falhas: Tópicos de Engenharia de Confiabilidade. 2004. Apostila. Curso de Engenharia de Manutenção Central.

[10] STAMATIS, D. H. Failure Modes and Effect Analysis: FMEA from Theory to Execution. $2^{\mathrm{a}}$ Edição. Milwalkee, Winsconsin: ASQ Quality Press, 2003.

[11] HELMAN, Horacio. ANDERY, Paulo Roberto Pereira. Análise de Falhas (Aplicação dos Métodosde FMEA - FTA). TQC: Gestão Pela Qualidade Total - Série Ferramentas da Qualidade, vol.11. 1ª Edição. Belo Horizonte: QFCO, 1995.

[12] SIQUEIRA. I. Patriota de. Manutenção centrada na confiabilidade. Editora Qualitymark, Rio de Janeiro, 2005. 